\title{
Physical activity frequency, independent of volume, is directly associated with C-reactive protein: NHANES 2003- 2006
}

GEOFFREY P. WHITFIELD'; WILLIAM E. KRAUS3, AND HAROLD W. KOHL III'1,2
${ }^{1}$ University of Texas Health Science Center; School of Public Health ${ }^{1,2}$ University of
Texas at Austin; Austin, TX 78701 ; ${ }^{3}$ Duke University School of Medicine, Durham, NC 27710.

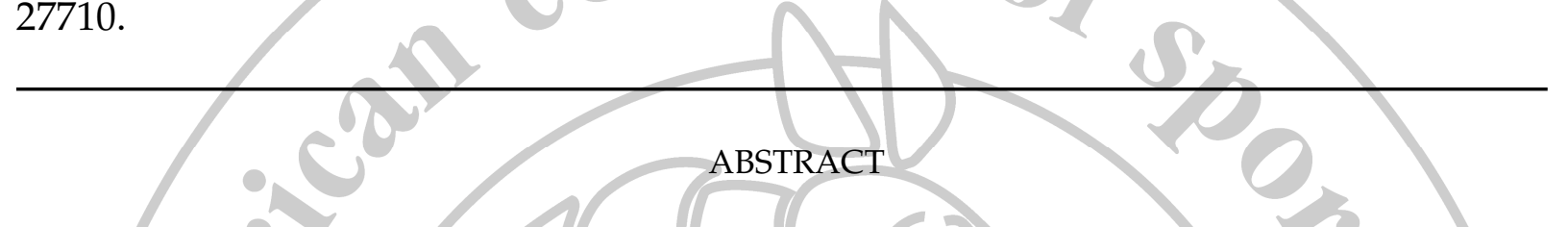

Physical activity (PA) has shown consistent positive effects on cardiovascular risk, yet the individual contributions of frequency, intensity and duration remain unclear. The purpose of this investigation was to explore the associations between objectively-measured PA frequency and cardiometabolic risk factors, independent of total PA volume. Data were analyzed for 5,549 adults with at least four valid days of accelerometry from the 2003 - 2006 US National Health and Nutrition Examination Survey (NHANES). Meeting PA guidelines was defined as 150+ minutes of moderate- to vigorous-intensity PA per week, accumulated in $10+$ minute bouts. Frequency was defined as the number of days containing at least one bout, and categorized as 1-3, 4-5, or 6-7 days per week $\left(\mathrm{d} \cdot \mathrm{wk}^{-1}\right)$. Risk factors were described for frequency categories after direct age adjustment and stratification by total weekly duration. Multivariate linear regression was used to analyze the independent associations between risk factors and PA frequency. Among those meeting PA guidelines, people in the lower total volume stratum and with higher frequency (4-5 or 6-7 $\left.\mathrm{d} \cdot \mathrm{wk}^{-1}\right)$ showed significantly higher C-reactive protein (CRP) compared to those in the lowest frequency category $\left(1-3 \mathrm{~d} \cdot \mathrm{wk}^{-1}\right)(0.12$ and $0.14 \mathrm{mmol} / \mathrm{L}$ vs. $0.08 \mathrm{mmol} / \mathrm{L}$, respectively, $\mathrm{p}<$ 0.05). Similar results were seen in the higher duration stratum, though differences in CRP between frequency categories did not reach significance. Small differences in hemoglobin-A1c and HDL-c were seen between frequency categories at both levels of duration $(\mathrm{p}<0.05)$. After adjustment for multiple confounders, a significantly higher CRP was seen in those accruing recommended PA on $4-5$ versus $1-3$ $\mathrm{d} \cdot \mathrm{wk}^{-1}(+40.3 \%, \mathrm{p}=0.024)$ and $6-7$ versus $1-3 \mathrm{~d} \cdot \mathrm{wk}^{-1}(+39.0 \%, \mathrm{p}=0.018)$. Controlling for total volume, moderate- to vigorous-intensity PA done on more days per week appears to be associated with higher CRP. This may be due to accumulation of CRP between bouts of PA when insufficient time is available for full clearance. This may decrease the prognostic value of CRP among frequently active subjects, and may offer an explanation for divergent findings between experimental and observational studies on the PA - CRP relationship.

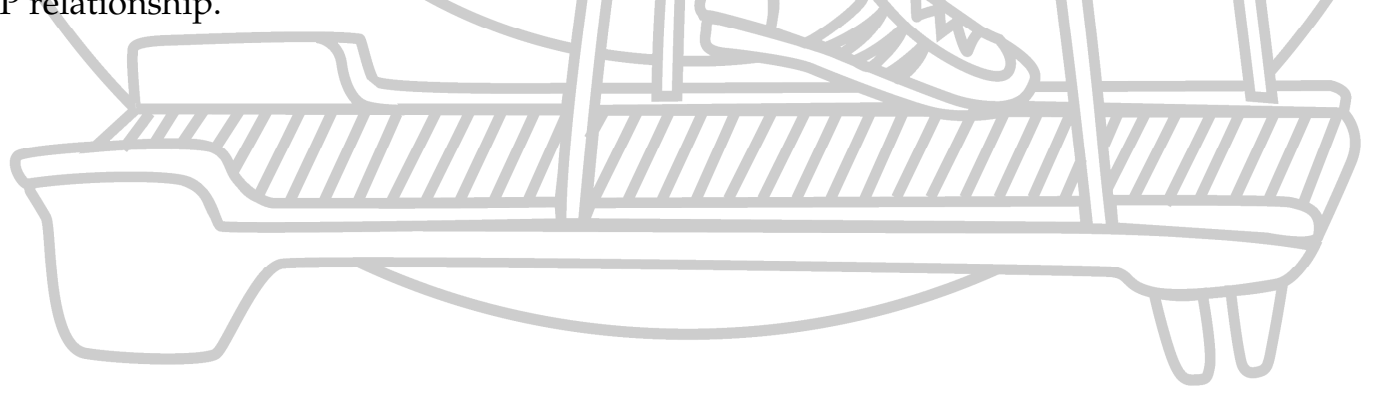




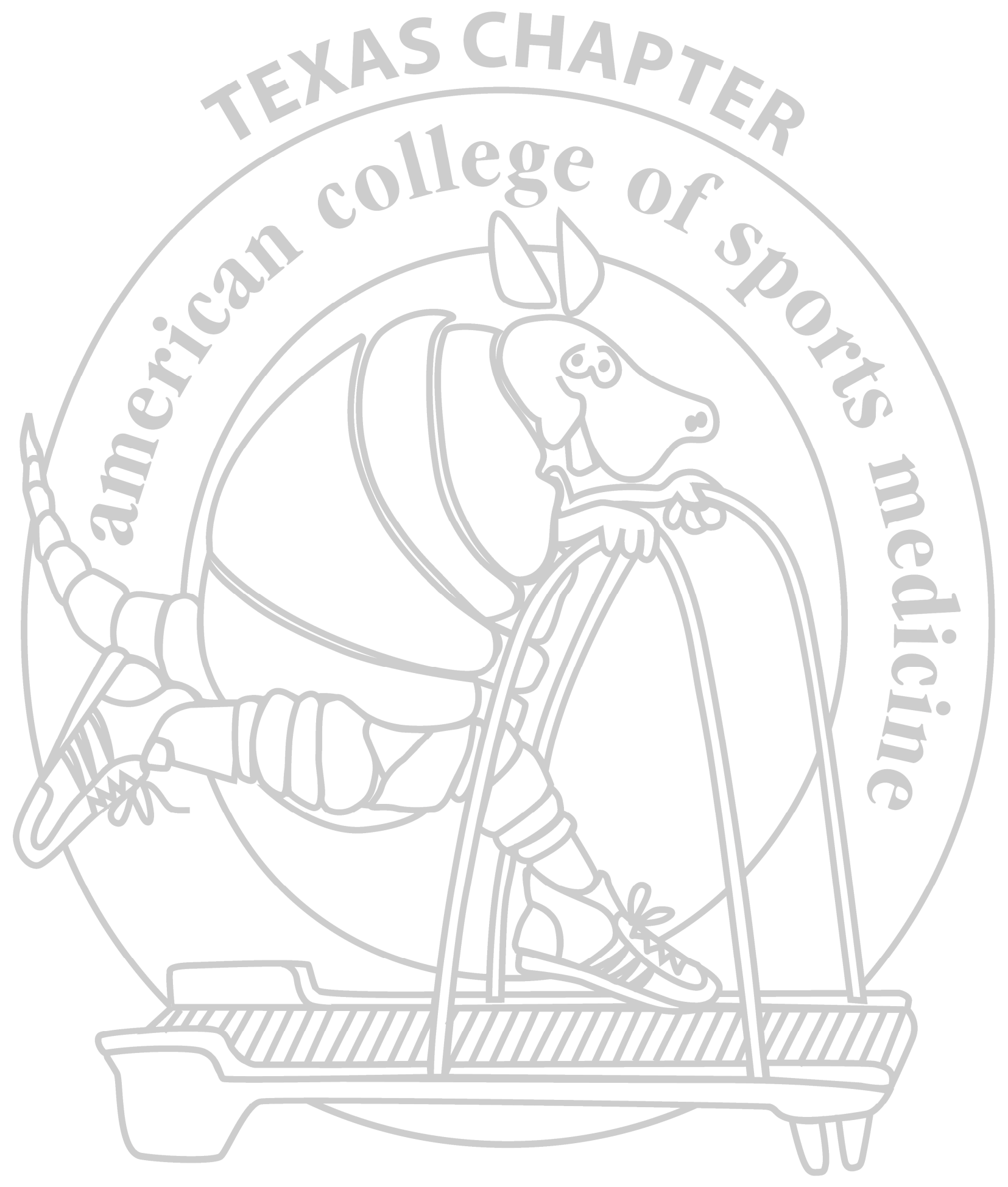

International Journal of Exercise Science

www.tacsm.org 